\title{
Development of the physical insight into the nature of the factors that control electrophysical and other properties of semiconductors
}

\author{
P.I. Baranskii, V.M. Babich, E.F. Venger \\ Institute of Semiconductor Physics, NAS Ukraine, 45 Prospect Nauki, 03028 Kyiv, Ukraine \\ Tel.: (380-44)265-62-80; Fax: (380-44)265-83-42;E-mail: journal@isp.kiev.ua
}

\begin{abstract}
Evolution of the physical insight into the nature of main factors that influence the energy band structure formation in semiconductors, and, hence, their basic electrophysical, optical, thermoelectrical and even mechanical properties, is reviewed. These factors are:

- dopant and residual impurities, intrinsic point defects, elongated defects (of the dislocation type),

- electrically active thermal donors and other complexes that are formed due to direct impurity-impurity or impurity-defect interaction.
\end{abstract}

Keywords: electron transport, point defect complex, impurity-impurity interaction, impurity-defect interaction.

Paper received 06.07.00; revised manuscript received 26.01.01; accepted for publication16.02.01.

\section{Introduction}

Analyzing wide variety of experimental results (especially, the ones of the magnetic resistance measurements for $n$-Ge and $n$-Si single crystals of known crystallographic orientations) available in the late 1950-ies, theoreticians gave up the description of the surfaces of equal energies in semiconductors by the regular spheroids. The shape of the isoenergetic surfaces was accepted to be the ellipsoids of rotation. The similar transformation of concepts occu-red in understanding of impurities distribution in the crystals. Physicists realized that in the crystal atoms of dopants and residual impurities do not necessarily exist only in the atomically-dispersed state.

Each stage of the historical evolution of concepts brought its own achievements, every following step did not ignore the previous one but used it as a scientific background that was further on supplemented by new experimental facts, and as a result the better understanding was gained.

Here we present the brief analysis of the most important achievements of the main stages of this evolution.

\section{Creation of the microscopic theory of electron transport in many-valley semiconductors (anisotropic scattering theory) and its experimental validation}

The first important step in the framework of the concept of atomically-dispersed impurity complexes in semiconductors was the creation of the theory of electronic transport in semiconductors [1,2] that took into account anisotropy of effective masses while the scattering processes were described using a scalar relaxation time.

More general approach (with the account of the anisotropy both of effective mass and relaxation time) was proposed in the well-known work by Herring [3]. However, the results of the above paper, as it was shown in [4] and [5], are valid only for the analysis of carriers scattering on the lattice vibrations.

Comprehensive and consistent development of the theory of anisotropic scattering (TAS) has been done by A.G. Samojlovich, P.M. Tomchuk, I.V. Dakhovskij, I.S. Buda, I.Ya. Korenblit, S.L. Korolyuk, L.I. Anatychuk, O.Ya. Luste and many other native and foreign scientists (see the original papers [6-8], as well as books $[5,9]$ and the references therein). 
Application of TAS for the analysis of the experimental results on electrophysical, galvanomagnetic, thermoelectric and thermomagnetic phenomena that was carried out by the scientists of ISP NASU demonstrated that many of the above-mentioned phenomena are essentially modified as compared to the conditions of isotropic scattering. The anisotropic scattering changes essentially the temperature and concentration dependencies of the kinetic coefficients, field dependencies, and dependencies on the angles between the vectors $\vec{\gamma}$ and $\vec{H}$, as well as on the direction of the uniaxial elastic deformation.

In [5] the theory of electrical and galvanomagnetic phenomena is thoroughly described and detailed comparison of the experimental results with theoretical predictions is carried out.

Description of the tensors $\hat{\rho}$ and $\hat{\alpha}$ by the sums of symmetric and antisymmetric parts gave the possibility to introduce the convenient classification of all possible longitudinal and transverse, odd and even as to magnetic field galvano- and thermomagnetic effects that appear in anisotropic media. The classification proposed evidences that besides the known phenomena some new effects should occur in anisotropic media that have never been studied before.

Investigations of $n$-Si and $n$-Ge resistivity variations under uniaxial elastic strain revealed several new mechanisms of piezoresistance having nothing in common with the well-known Smith-Herring mechanism of piezoresistance that is caused by carriers redistribution between isoenergetic ellipsoids which are no longer degenerate due to uniaxial strain.

Detailed comparison of the experimental magnetic field dependencies of Hall coefficient with dependence $R_{H}=f(H)$ calculated in the framework of TAS have shown that in many-valley semiconductors (like $n$-Si and $n$-Ge) the Hall coefficient remains anisotropic in the range of classically high $H$, i.e. the Hall-factor $r$ is not equal to unity in the whole range of magnetic field variation (up to the range where quantum effects are observed).

For the first time the consistent microtheory of thermoelectric and thermomagnetic phenomena in anisotropic crystals at phonon-drag of electrons was developed in [10] including the case of uniaxial stress arbitrarily oriented as to the crystallographic axes. Thus, TAS was applied to the new wide class of phenomena occurring in anisotropic semiconductors in electric and magnetic fields, under elastic strain and in the presence of temperature gradients.

Only two parameters that can be experimentally measured are necessary for qualitative and quantitative description of the variety of the above phenomena. These are the mobility anisotropy $K=\mu_{\perp} / \mu_{\text {II }}$ and anisotropy of thermoe.m.f. of the phonon drag of electrons $M=$ $=\alpha_{\text {II }}^{\Phi} / \alpha_{\perp}^{\Phi}$; these two parameters characterize a separate isoenergetic ellipsoid.

Application of uniaxial elastic deformation (in wide range of mechanical strains $\mathrm{X}$ ) provided the possibility to analyze the above phenomena under the conditions when different number of the isoenergetic ellipsoids is involved (from one ellipsoid in the case of $<111>$ strain in $n$-Ge to six ellipsoids in unstrained $n$-Si).

The experimental data obtained during the last three decades in our country and abroad dealing with the investigations of thermo-e.m.f. in many-valley semiconductors are analyzed in [11] and the comparison with theoretical calculations is carried out. The authors made an attempt to generalize experimental and theoretical data on the thermoelectric and thermomagnetic phenomena in many-valley semiconductors (like $n-\mathrm{Ge}$ and $n$-Si) under the conditions of phonon drag of electrons. Both undeformed and uniaxially deformed cases as well as influence of arbitrarily directed magnetic fields in the nonquantizing magnetic field range were analyzed.

TAS was generalized for the case of quantizing magnetic fields in $[12,13]$, and the results of the experiments pretty well agree with the theoretical predictions.

\section{Results on the impurity-impurity and impurity-defect interaction study}

During the development and optimization of the technology of growth of Si and Ge single crystals (by Czochralski method) the change of the ingots resistivity under annealing was demonstrated. The change is caused by the appearance of electrically active thermal donors that are formed in the crystal from the residual oxygen impurity. As the technology of Si- and Ge-based devices implies a series of procedures involving annealings (that differ by temperature and duration) this property of oxygen impurity in $\mathrm{Cz}-\mathrm{Si}$ and $\mathrm{Ge}$ was thoroughly reviewed by the authors of [14] who gathered, systematized and generalized wide variety of experimental and theoretical data on oxygen behavior in silicon. The sources of oxygen in silicon, properties of atomical oxygen and mechanisms of defects formation in oxygen-rich silicon in wide temperature range $\left(400-1300^{\circ} \mathrm{C}\right)$ are discussed in details; existing models of the oxygen-related electrically active complexes (thermal donors) are also analyzed. The influence of dopants and background impurities (including carbon) on the kinetics of the formation, accumulation and thermal annealing as well as other properties of thermal donors-I and thermal donors-II in silicon crystals are described.

Modern technology of silicon growth provides two types of crystals - with low oxygen concentration, $N_{\mathrm{O}} \leq$ $\leq 3 \cdot 10^{16} \mathrm{~cm}^{-3}$, that are grown by floating zone method and with high oxygen concentration, $N_{\mathrm{O}} \leq(2-10) \cdot 10^{17} \mathrm{~cm}^{-3}$, that are grown by Czochralski method. The silicon crystals with oxygen content in the intermediate range (from $3 \cdot 10^{16} \mathrm{~cm}^{-3}$ to $2 \cdot 10^{17} \mathrm{~cm}^{-3}$ ) are not available, while they can be useful (in accordance with certain predictions) for microdevice production.

\section{Characteristic features of electrically active complexes produced by radiation}

According to the authors of [15], extremely high mobility of interstitials (down to $T \sim 0.5-1.5 \mathrm{~K}$ ) and vacancies 


\section{P.I. Baranskii et al.: Development of the physical insight into the factors ...}

and, hence, high probability of their interaction cause the preferable formation of complexes (or associates) of radiative defects. Examples of vacancy-related complexes are a vacancy plus donor (E-center) or a vacancy plus oxygen atom (A-center). On base of the analysis carried out in [15] the conclusion was made that the stability of radiative defects increases with the number of structural units that are involved in the complex, and hence, with the increase of the complex size.

In [16] it is shown that the annealing of any defect (or complex) depends both on its own energy parameters and on the parameters of other defects that take part in the process.

Kinetics of electronic processes in crystals subjected to various types of penetrating radiation has been widely investigated; the results obtained are reviewed in the doctorate thesises [17] and [18]. Special attention was paid to the changes of the energy positions of electrically active centers in uniaxially elastically deformed crystals $n$-Si and $n$-Ge. The influence of the growth layers (strates) that correspond to inhomogeneous (almost periodic) distribution of doping and residual impurities along the direction of crystal growth on the anisotropy of carriers scattering and other properties of the crystals was studied as well.

Another important semiconductor material (along with $\mathrm{Si}$ and $\mathrm{Ge}$ ) is GaAs. Its electrophysical properties and radiative stability are also studied in the department \#7 (in collaboration with other departments of the ISP NASU). In the book by A. Ye. Belyaev, R. V. Konakova, I. V. Prokopenko et. al. [19] the effect of radiation on GaAs and GaAs-based devices is reviewed. Mechanisms of defects formation under the radiation and radiation annealings are discussed. Methods of improvement of radiative stability of GaAs-based devices are analyzed on base of the scientific papers published during 19851996. The above review will be useful for the design of GaAs-based devices for operation under high radiation level conditions both on the Earth and in the outer space.

\section{Conclusions}

1. Complex study of n-Si and n-Ge single crystals have proved that the overwhelming majority of electrophysical phenomena in these crystals are caused rather by the complexes of point defects than by point defects themselves; thus the behavior of semiconductors essentially changes in electrical and magnetic fields, under mechanical strain, and, especially, at the irradiation of the semiconductors with penetrating radiation when radiation defects are formed and radiation annealing takes place.

2. The changes that occur due to interdefect (or impurity-defect) interaction should be taken into account in the development of various semiconductor devices, especially when annealings (or even series of consequent annealings) are inevitably involved into the technological process.

3. In view of the diminishing of device sizes and the transition from micrometer and sub-micrometer scale to nanoscale, e.g. in superlattices and nanostructures, the role of interdefect interaction becomes even more important. Thus, both theoretical and experimental basis for the analysis of interdefect interaction in the new range of scales should be developed. At the above conditions the physics of the processes will be similar to the phenomena observed in porous silicon [20] or in nanocomposite films [21].

4. Deep understanding of interatomic (interdefect) interaction in semiconductors is important both for development of totally new principles of device operation and for achievement of reliable and stable operation of the devices in rather difficult (and often extremal) conditions.

\section{References}

1. B. Abeles, S. Meiboom, Theory of the Galvanomagnetic Effects in Germanium // Phys. Rev. 1, pp.31-37 (1954).

2. M. Shibuya, Magnetoresistance Effect in Cubic Semiconductors with Spheroidal Energy Surfaces // Phys. Rev. 95(6), pp. 1385-1393 (1954).

3. C. Herring, Transport and Deformation-Potential Theory for Many-valley Semiconductors // Phys.Rev. 101(3), pp. 944962 (1995).

4. P.I. Baranskii, Theoretical and experimental investigations of the transport phenomena in many-valley semiconductors under extremal conditions, in Fundamentals of the semiconductor electronics, Ed. O. V. Snitko, pp. 161-172, Kiev, «Naukova dumka» (1985).

5. P.I. Baranskii, I.S. Buda, I.V. Dakhovskii et. al., in Electrical and galvanomagnetic phenomena in anisotropic semiconductors, Ed. «Naukova dumka», p. 270 (1977).

6. A.G. Samoilovich, I.S. Buda, I.V. Dakhovskii, Theory of anisotropic scattering //Fizika $i$ Tekhnika poluprovodnikov 7(4), p. 859 (1973).

7. A.G. Samoilovich, I.Ya. Korenblit, I.V. Dahovskii, V.D. Iskra, Solution of the kinetic equation at the anisotropic scattering of electrons // Fizika i Tekhnika poluprovodnikov 3(10), pp. 2939-2952 (1961).

8. A.G. Samoilovich, I.Ya. Korenblit, I.V. Dakhovskii, V.D. Iskra, Anisotropic scattering of electrons by ionized impurities and acoustic phonons // Fizika Tverdogo Tela 3(11), pp. 32853298 (1961).

9. I.M. Dykman, P.M. Tomchuk, Transport phenomena and fluctuations in semiconductors, Ed. «Naukova dumka», p. 320, Kiev (1981).

10. P.I. Baranskii, I.S. Buda, I.V. Dakhovskii, Theory of thermoelctric and thermomagnetic phenomena in anisotropic semiconductors, Ed. «Naukova dumka», p.272, Kiev (1987).

11. P.I. Baranskii, I.S. Buda, V.V. Savyak, Thermoelctric and thermomagnetic phenomena in many-valley semiconductors, Ed. "Naukova dumka», p.269, Kiev (1992).

12. P.I. Baranskii, S.L. Korolyuk, A.G. Samoilovich et al., Longitudinal Magnetoresistance of $n$-Type Germanium in the Quantum Limit // Phys. Stat. Sol. (b) 60, pp.203-211 (1973).

13. V.M. Babich, P.I. Baranskii, A.G. Samoilovich et al., Transverse Magnetoresistance of $n$-Ge in Quantizing Magnetic Fields // Phys. Stat. Sol. (b) 55, pp.457-466 (1973).

14. V.M. Babich, N.I. Bletskan, E.F. Venger, Oxygen in single crystalline silicon, Ed. "Interpres Ltd.», p.240, Kiev (1997).

15. Problems of radiation technology of semiconductors, Ed. L. S. Smirnov, «Nauka», p.294, Novosibirsk (1980).

16. Physical processes in the irradiated semiconductors, Ed. L. S. Smirnov, «Nauka», p.256, Novosibirsk (1977).

17. A.V. Fedosov, Physical basis of tensoeffects in many-valley semiconductors under extremal conditions, Doctorate thesis, Kiev, pp.348 (1985). 
P.I. Baranskii et al.: Development of the physical insight into the factors ...

18. A.K. Semenyuk, Electronic properties of many-valley semiconductors with deep radiation centers, Doctorate thesis, Lutsk, pp.387 (1993).

19. A.E. Belyaev, E.F. Venger, M. Veseli et al., Radiative stability of ultrahigh frequency devices with Shottki barrier (Some physical and technical aspects), Ed. «Interpres Ltd.», p.128, Kiev (1998).
20. S.V. Svechnikov, E.B. Kaganovich, E.G. Manojlov, V.S. Dvirnyak, Visible electroluminescence of porous silicon structures (review) // Optoelectronics and semiconductors technique 32, pp. 19-49 (1997).

21. S.V. Svechnikov, E.B. Kaganovich, E.G. Manojlov, V.S. Dvirnyak, Electroluminescence of $\mathrm{Si}$ nanocomposite films // Optoelectronics and semiconductors technique 33, pp. 3-14 (1998). 